\title{
An analysis of the hybrid FDTD scheme for modeling the propagation of the electromagnetic waves in a cold magnetized toroidal plasma
}

\author{
Maryna Surkova ${ }^{1}$, Guido Van Oost ${ }^{1}$, Yvan Pavlenko ${ }^{2}$, Dirk Van Eester ${ }^{3}$ and Daniël De Zutter ${ }^{4}$ \\ ${ }^{1}$ Department of Applied Physics, Ghent University Ghent, Sint- Pietersnieuwstraat 41 B4, 9000 Ghent, \\ Belgium \\ ${ }^{2}$ Department of Physics and Technology, Kharkiv Karazin National University, Svobody sq. 4, 61077 \\ Kharkiv, Ukraine \\ ${ }^{3}$ Laboratory for Plasma Physics- ERM/KMS, "Association EUROATOM- Belgia State", Renaissancelaan \\ 30 Avenue de la Renaissance, B- 1000 Brussels, Belgium \\ ${ }^{4}$ Electromagnetic Group, Department of Information Technology, Ghent University, Sint-
}

Pietersnieuwstraat 41, 9000 Ghent, Belgium

\begin{abstract}
To explore the behavior of electromagnetic waves in cold magnetized plasma, a three-dimensional cylindrical hybrid finite-difference time-domain (FDTD) model is developed. The full discrete dispersion relation is derived and compared to the exact solutions. We establish an analytical proof of stability in the case of nonmagnetized plasma. We demonstrate that in the case of nonmagnetized cold plasma the maximum stable Courant number of the hybrid method coincides with the vacuum Courant condition. In the case of magnetized plasma the stability of the applied numerical scheme is investigated by numerical simulation. In order to determine the utility of the applied difference scheme we complete the analysis of the numerical method demonstrating the limit of the reliability of the numerical results.
\end{abstract}

\footnotetext{
${ }^{1}$ Email address: Maryna.Surkova@ugent.be
} 


\section{Introduction}

A lot of research effort has been spent over many years to improve FDTD methods to model wave propagation in many different types of media, in particular in non-magnetized and magnetized plasma. A thorough overview of methods to model isotropic cold plasma can be found in [1]. For more recent efforts on magnetized plasma we refer to the work of Smithe [2], and enhancements along the lines of Smithe in [3], and [4]. The methods proposed in [2] and [3] are hybrid methods as Faraday's law is descretized explicitly (classical FDTD scheme) and Ampere's law implicitly. The method of [4] is the fully implicit method.

In this work, we construct the three-dimentional FDTD model in order to describe electromagnetic wave propagation in a magnetized toroidal plasma. In order to examine the efficiency of the applied numerical scheme the analysis of the reliability of the numerical results is necessary. Therefore a rigorous stability analysis is provided. It is shown that the maximum stable Courant number in non-magnetized cold plasma is unity just as for free space [1]. In the case of magnetized plasma the complexity of the implemented numerical model does not allow to apply a standard analytical analysis that has been discussed in [5]. However, the problem is investigated numerically using the von Neumann method [6].

\section{Basic equations}

Including the driven equation of motion, Maxwell's equations in the three-dimensional case describing cold magnetized plasma in time-domain are:

$$
\begin{gathered}
\frac{\partial \mathbf{B}}{\partial t}=-\nabla \times \mathbf{E} \\
\epsilon_{0} \frac{\partial \mathbf{E}}{\partial t}=-\sum_{s} \mathbf{J}_{s}+\nabla \times \mathbf{H}, \\
\left(\frac{\partial}{\partial t}+\nu_{s}\right) \mathbf{J}_{s}=\epsilon_{0} \omega_{p s}^{2} \mathbf{E}-\boldsymbol{\Omega}_{s} \times \mathbf{J}_{s} .
\end{gathered}
$$

Here, the subscript $s$ denotes the charged particle species in the plasma ( $e, i$ for electrons and ions respectively). $\mathbf{E}$ is the electric field vector $(V / m), \mathbf{B}=\mu_{0} \mathbf{H}$ is the magnetic flux density vector $\left(W b / m^{2}\right), \mathbf{H}$ is the magnetic field vector $(A / m), \mathbf{J}_{s}$ is the current density vector of a particular specie $\left(A / m^{2}\right)$. And $\nu_{s}$ is a collision frequency term. The vacuum permeability $\mu_{0}(H / m)$ and vacuum permittivity $\epsilon_{0}(F / m)$ are independent of the frequency. The plasma frequency $\omega_{p s}(\mathrm{rad} / \mathrm{sec})$ is defined as

$$
\omega_{p s}=\sqrt{\frac{n_{s} q_{s}^{2}}{m_{s} \epsilon_{0}}}
$$

where $n_{s}\left(1 / \mathrm{m}^{3}\right)$, is the density, $q_{s}(C)$, is the charge and $m_{s}(\mathrm{~kg})$, is the mass of a given specie. Further, by construction the cyclotron frequency is

$$
\Omega_{s}=\frac{q_{s} B_{0}}{m_{s}},
$$

where $B_{0}$ is the background magnetic field oriented along the $\varphi$-axis. 


\section{Time Domain and Space Discretization}

The set of equations (1-3) is applied to the FDTD mesh (See Fig. 1) [7]. Here, the E-field and the current density $\mathbf{J}$-field are defined at integer time steps while the $\mathbf{H}$-field is defined at half-integer time steps [2]. The resulting approximations are:

$$
\begin{aligned}
\mu_{0}\left[\frac{\mathbf{H}^{n+1 / 2}-\mathbf{H}^{n-1 / 2}}{\Delta t}\right] & =-\nabla \times \mathbf{E}^{n}, \\
\epsilon_{0}\left[\frac{\mathbf{E}^{n+1}-\mathbf{E}^{n}}{\Delta t}\right]+\left[\frac{\mathbf{J}_{s}^{n+1}+\mathbf{J}_{s}^{n}}{2}\right] & =\nabla \times \mathbf{H}^{n+1 / 2}, \\
{\left[\frac{\mathbf{J}_{s}^{n+1}-\mathbf{J}_{s}^{n}}{\Delta t}\right]+\nu_{s}\left[\frac{\mathbf{J}_{s}^{n+1}+\mathbf{J}_{s}^{n}}{2}\right]=} & \epsilon_{0} \omega_{p s}^{2}\left[\frac{\mathbf{E}^{n+1}+\mathbf{E}^{n}}{2}\right] \\
& -\left[\Omega_{s} \times \frac{\mathbf{J}_{s}^{n+1}+\mathbf{J}_{s}^{n}}{2}\right] .
\end{aligned}
$$

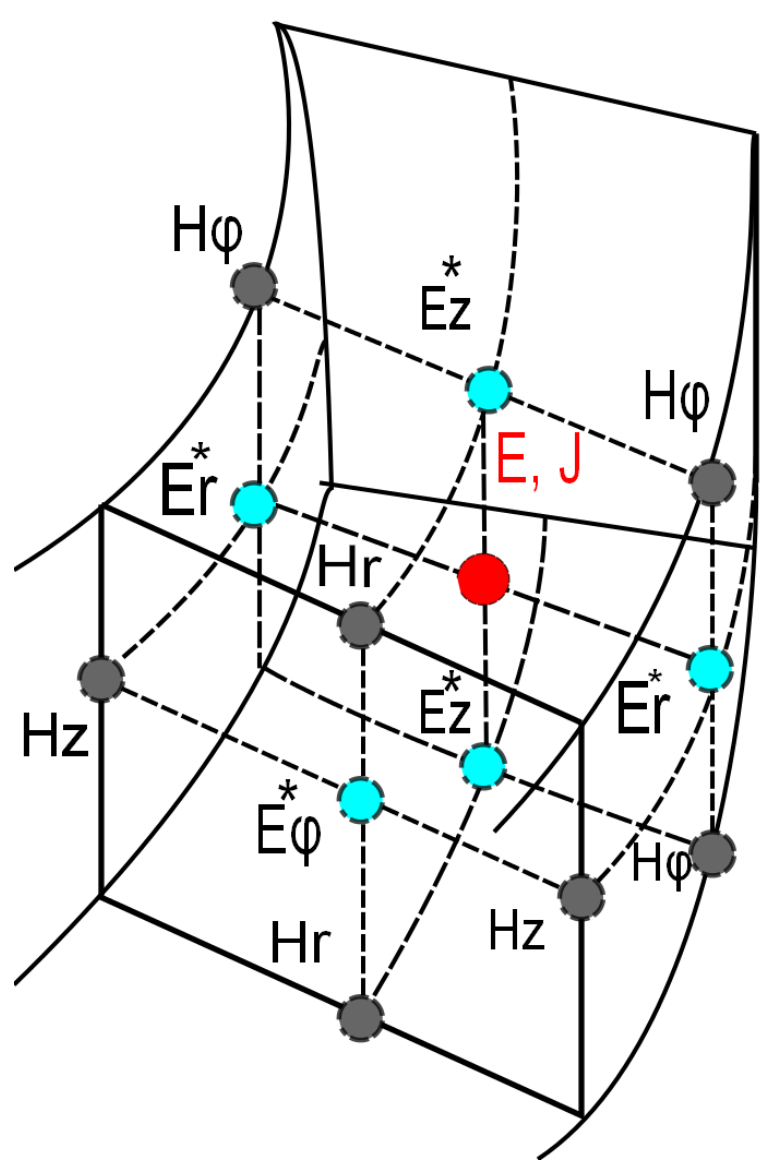

Figure 1: Yee cell. The components $H_{r}, H_{\varphi}, H_{z}$ of the magnetic field $\mathbf{H}$ are localized at the center of the edges of the Yee- cell. The method proposed here initially locates the $J_{r}, J_{\varphi}$, and $J_{z}$ components at the same positions as the $E_{r}, E_{\varphi}$, and $E_{z}$ components : at the center of the Yee- cell. It has to be noted that the spatial averaging is required to calcultate the central- difference derivatives. The averaged values of the electric field components are marked with a star $(*)$. 


\section{Full Discrete Dispersion Relation}

In this section the fully discretized dispersion relation $f(k, \omega)=0$ is derived. Since the eigenfunctions of the wave equation in cylindrical coordinate system cannot be presented as a plane wave with definite $k$ and $\omega$, the dispersion relation is obtained in the Cartsian coordinates. The wave fields and plasma currents are expanded as a Fourier series in space and time (9).

$$
\mathbf{E}, \mathbf{H}, \mathbf{J}_{s} \propto \exp \left(j\left(k_{x} x+k_{y} y+k_{z} z-\omega t\right)\right)
$$

If the wavelength is significanly shorter than the distance to the cylindrical axis, the Cartesian dispersion relation approximates the cylindrical one, therefore the full discrete dispersion relation is:

$$
\begin{aligned}
& \left(a_{1}^{2} a_{2}^{2} b_{3}^{2} \epsilon_{1}+a_{1}^{2} a_{3}^{2} b_{2}^{2} \epsilon_{3}+a_{2}^{2} a_{3}^{2} b_{1}^{2} \epsilon_{1}\right) \tilde{k}^{2} \\
& -\left(a_{1}^{2}\left(b_{2}^{2}+b_{3}^{2}\right) \epsilon_{1} \epsilon_{3}+a_{2}^{2}\left(b_{1}^{2}+b_{3}^{2}\right)\left(\epsilon_{1}^{2}-\epsilon_{2}^{2}\right)+a_{3}^{2}\left(b_{1}^{2}+b_{2}^{2}\right) \epsilon_{1} \epsilon_{3}\right) b_{4}^{2} \\
& +\epsilon_{3}\left(\epsilon_{1}^{2}-\epsilon_{2}^{2}\right) b_{4}^{4}=0,
\end{aligned}
$$

where

$$
\begin{gathered}
\epsilon_{1}=1-\sum_{\alpha} \frac{\omega_{p \alpha}^{2} \tilde{\omega}}{\omega^{*}\left(\tilde{\omega}^{2}-\omega_{\alpha \varphi}^{2}\right)}, \\
\epsilon_{2}=-\sum_{\alpha} \frac{\omega_{p \alpha}^{2} \omega_{\alpha \varphi}}{\omega^{*}\left(\tilde{\omega}^{2}-\omega_{\alpha \varphi}^{2}\right)}, \\
\epsilon_{3}=1-\sum_{\alpha} \frac{\omega_{p \alpha}^{2}}{\omega^{*} \tilde{\omega}}, \\
\omega^{*}=\frac{2}{\Delta t} \tan (\omega \Delta t / 2), \\
\tilde{\omega}=\omega^{*}+i \nu_{s}, \\
a_{1} \equiv \cos \left(k_{r} \Delta r / 2\right), \quad b_{1} \equiv \frac{2}{\Delta r} \sin \left(k_{r} \Delta r / 2\right), \\
a_{2} \equiv \cos (n \Delta \varphi / 2), \quad b_{2} \equiv \frac{2}{r \Delta \varphi} \sin (n \Delta \varphi / 2), \\
a_{3} \equiv \cos \left(k_{z} \Delta z / 2\right), \quad b_{3} \equiv \frac{2}{\Delta z} \sin \left(k_{z} \Delta z / 2\right), \\
a_{4} \equiv \cos (\omega \Delta t / 2), \quad b_{4} \equiv \frac{2}{c \Delta t} \sin (\omega \Delta t / 2),
\end{gathered}
$$

and $\tilde{k}^{2} \equiv b_{1}^{2}+b_{2}^{2}+b_{3}^{2}$ is a square of the discretized wave vector.

\subsection{Vacuum case}

In vacuum case when the plasma density is zero, the discrete dispersion relation becomes:

$$
k_{r}^{2}+(n / r)^{2}+k_{z}^{2} \equiv k^{2}=\omega^{2} / c^{2} .
$$


Demanding that real $k$ always maps to real $\omega$ in (12) it requires the Courant condition:

$$
c \Delta t<\frac{1}{\sqrt{\left(\frac{1}{\Delta r}\right)^{2}+\left(\frac{1}{r \Delta \varphi}\right)^{2}+\left(\frac{1}{\Delta z}\right)^{2}}} .
$$

The expression (12) is a well-known dispersion relation for electromagnetic waves in vacuum described in [10], [11].

\subsection{Nonmagnetized case}

In the absence of a background magnetic field and if the collision frequency term is neglected, the full discrete dispersion relation transforms into the slow wave dispersion relation:

$$
k_{r}^{2}+(n / r)^{2}+k_{z}^{2} \equiv k^{2}=\epsilon_{3}\left(\omega^{2} / c^{2}\right)
$$

The analysis of the (14) shows that the Courant condition in case of a nonmagnetized plasma is absolutely equivalent to the vacuum Courant condition (13).

\subsection{Magnetized plasma}

In $1 \mathrm{D}$ case when the problem is uniform along the $z$ and $\varphi$ directions, the discrete dispersion relation is:

$$
\epsilon_{1} \tilde{k}^{4}-\left(\epsilon_{1} \epsilon_{3}+\left(\epsilon_{1}^{2}-\epsilon_{2}^{2}\right)\right) b_{4}^{2} \tilde{k}^{2}+\epsilon_{3}\left(\epsilon_{1}^{2}-\epsilon_{2}^{2}\right) b_{4}^{4}=0 .
$$

Equation (15) gives two well-known solutions. One of them is the slow wave dispersion relation (14) and the second one is the extraordinary wave [10]:

$$
k^{2}=\frac{\epsilon_{1}^{2}-\epsilon_{2}^{2}}{\epsilon_{1}} \frac{\omega^{2}}{c^{2}}=\alpha \frac{\omega^{2}}{c^{2}} .
$$

\section{Reliability of the Numerical Results}

The numerically obtained results should in principle reflect the results we get analytically. Both results however are not completely identical. There are several reasons for this difference. The impact of each of the reasons questions whether the applied numerical scheme is accurate enough. Usually the utility of the numerical scheme is determined by a stability test. One of the well-known stability requirements is the Courant condition. It requires the time step $\Delta t$ to have a specific bound on the grid step. For example, as it is shown above, the maximum stable Courant number in unmagnetized cold plasma is unity just as for the vacuum case. Though in case of magnetized plasma, due to the complexity of the applied numerical scheme the stability analysis is rather arduous. It has been found empirically that the stability requirement in case of non-magnetized plasma is still valid for the magnetized case [12]. There is however no analytical proof. We assume that when a high-density nonuniform plasma possesses one (or more) ion species, there exists

a plasma resonance area where it might be problematic to satisfy the Courant condition. Therefore in the following section we are going to determine the limit of the reliability of the numerical results. 


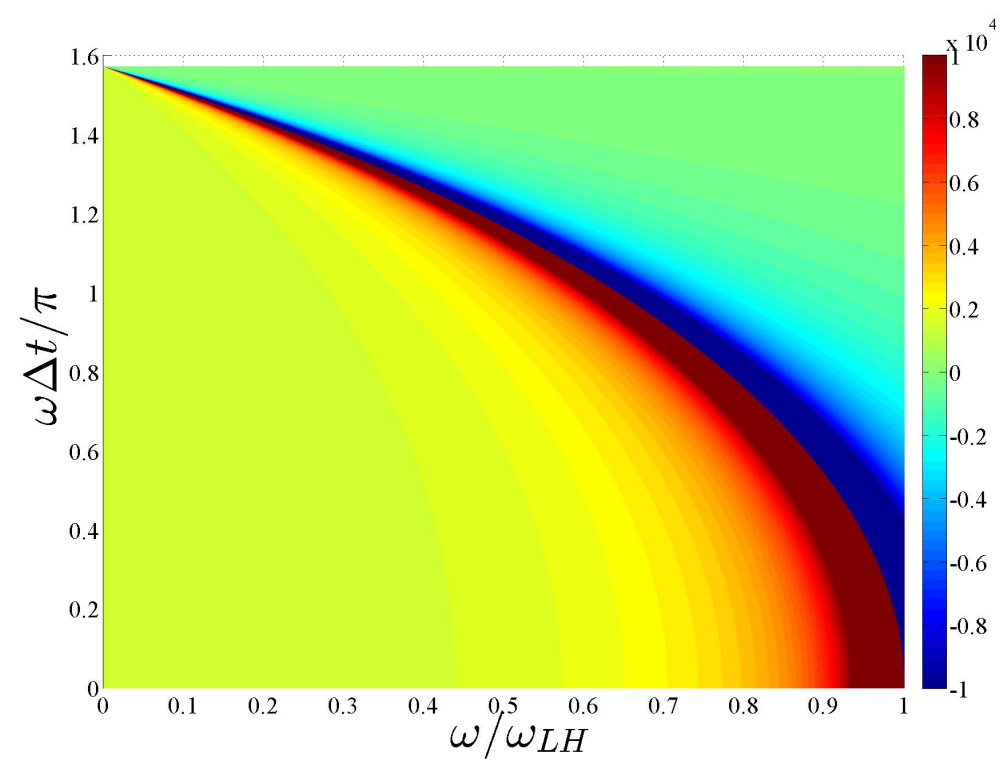

Figure 2: The numerical dispersion function of the extraordinary wave in the low frequency range in oneion (Deuterium) plasma media. The simulation parameters corresponding to this particular test are: the plasma electron density is equal to the deuterium (D) ion density $n_{e}=n_{D}=3 \cdot 10^{19}\left(1 / \mathrm{m}^{3}\right)$, the background magnetic field $B_{0}$ is equal to $3 \mathrm{~T}$. The time step $\Delta t$ is changing in order to provide the variation of the argument of the tangent of the numerical frequency from 0 to $\pi / 2$. In order to perform the sufficient spatial resolution, 10 points per wavelength are used to determine the cell size [8-9].

Here, we choose to simulate the positive part of the extraordinary wave (15) in a low frequency range. According to the analytical dispersion relation it spreads from 0 to the low hybrid frequency $\omega_{L H}=$ $\left(\left(\Omega_{e} \Omega_{i}\right)^{-1}+\omega_{p i}^{-2}\right)^{-1 / 2}$. If the numerical dispersion function appears to be negative in the range where the analytical dispersion function must be positive, the numerical scheme may be unstable or lead to poor numerical accuracy. Fig. (2) presents the results of the simulations. The figure shows that the dispersion numerical function acquires negative values (the blue area) near the lower hybrid resonance. For the sake of comparison, in Fig. (3) the upper limit of the time increment $\Delta t$ is plotted next to the vacuum Courant condition. According to Fig. (2) and Fig. (3) the numerical dispersion function becomes negative above the black dashed line thus limiting the reliability of the numerical results to the area below this (dashed) line. However, Fig. (3) shows that the vacuum Courant condition is definitely stronger than the upper limit of the time increment mentioned above. We would like the reader to pay attention to the area where the plasma resonance occurs. Fig. (3) shows that the time step $\Delta t$ tends towards zero exactly near the plasma resonance area, therefore it might be challenging to simulate in this range. As a practical example, the one dimensional problem of the fast wave mode propagating through a three component plasma (electron, deuterium (D) and hydrogen $(\mathrm{H})$ ions) with a nonuniform magnetic field is considered. In Fig. (4) the black line presents a typical wave dispersion in a cold collisionless plasma. The position of the plasma resonance (where the wave vector goes to infinity) and the plasma cut-off (where the wave vector becomes equal to zero) are also shown in the figure. On the left-hand side of the resonance, both the wave phase velocity and the wave length tend towards zero. In order to resolve the short wavelength on the left-hand side of the resonance the grid step should be much smaller than the wavelength $\left(\Delta r<<1 / k_{r}\right)$. On the other hand both the phase velocity and 


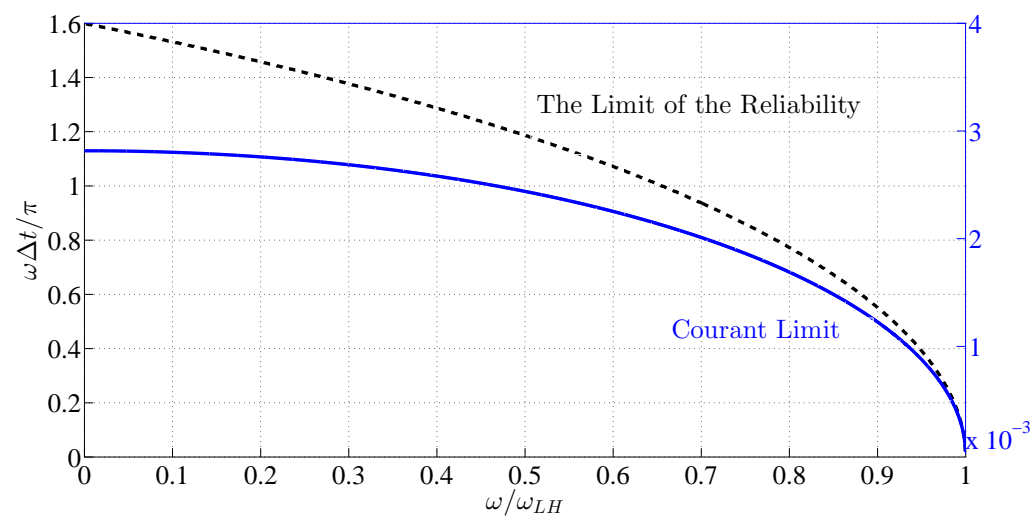

Figure 3: The limit of the reliability of the numerical scheme. The black dashed line shows the upper limit of the time increment of the applied FDTD algorithm in case of magnetized plasma. The blue curve is the vacuum Courant Condition.

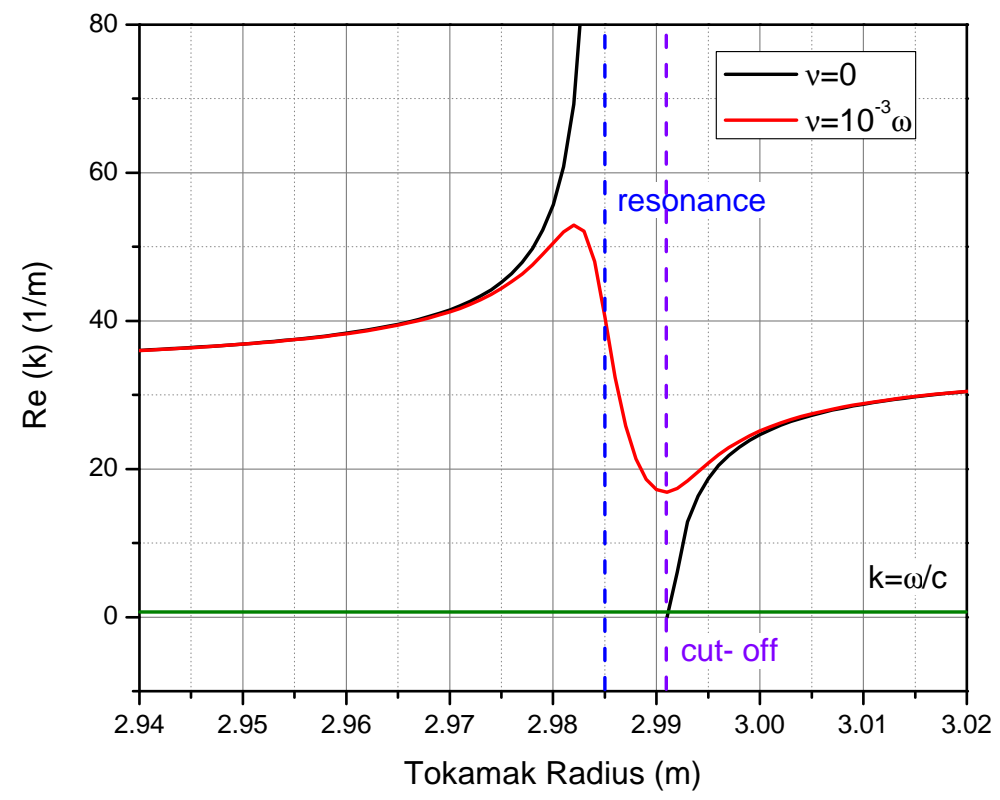

Figure 4: The dispersion of the fast wave propagation through the three component plasma in a nonuniform magnetic field.

the wavelength tend toward infinity near the plasma cut-off where the grid step cannot be larger than the plasma column resolution requirement $(\Delta r<<1 / a$, where $a$ is the width of a plasma region). According to the numerical example described above (see Fig.3), it is not feasable to comply with the vacuum Courant condition near the plasma resonance. Therefore the suitable grid step must be carefully chosen. The criteria for choosing the size of the grid step may be obtained through the analysis of the dispersion relation.

The next test is provided for the case where the collision frequency term is included. The red line in Fig.(4) shows the fast wave dispersion. The plasma resonance and cut-off have disappeared due to the presence of the collision frequency term $\left(\nu=10^{-3} \omega\right)$. The values of the wavelength are bounded from below 
and the phase velocity does not exceed the speed of light. Hence, the inclusion of the collision frequency term gives us the possibility to overcome the stability problem near the plasma resonance area. The collision frequency term certainly provides the additional power dissipation near the evanescent layer (the range between the resonance and the cut- off). But for this particular test the magnitude of the collision frequency term is chosen in such a way that the provided damping is negligible for the wave propagation outside the evanescent layer.

\section{Numerical Stability}

The numerical stability analysis can be provided based on the von Neumann method [6]. The 1D problem with the wave propagationg in the r-direction with non-zero components $E_{r}, E_{z}, H_{\varphi}, J_{r}, J_{z}$ in magnetized plasma is considered. The set of the difference equation can be written in the general form:

$$
\underbrace{F^{t+1}}_{\text {future }}=M \underbrace{F^{t}}_{\text {past }}
$$

where $F$ represents a column vector containing all discretized electric, magnetic and current field components, $M$ is an amplification matrix. The numerical scheme is stable if the eigenvalues lie on the unit circle in the complex plane. However, if there are any losses assumed either due to the nature of the material or due to the radiation phenomena, some of the eigenvalues may be inside the unit circle. In Fig.(5) the eigenvalues of the amplification matrix of the applied FDTD method in case of magnetized plasma are presented. The

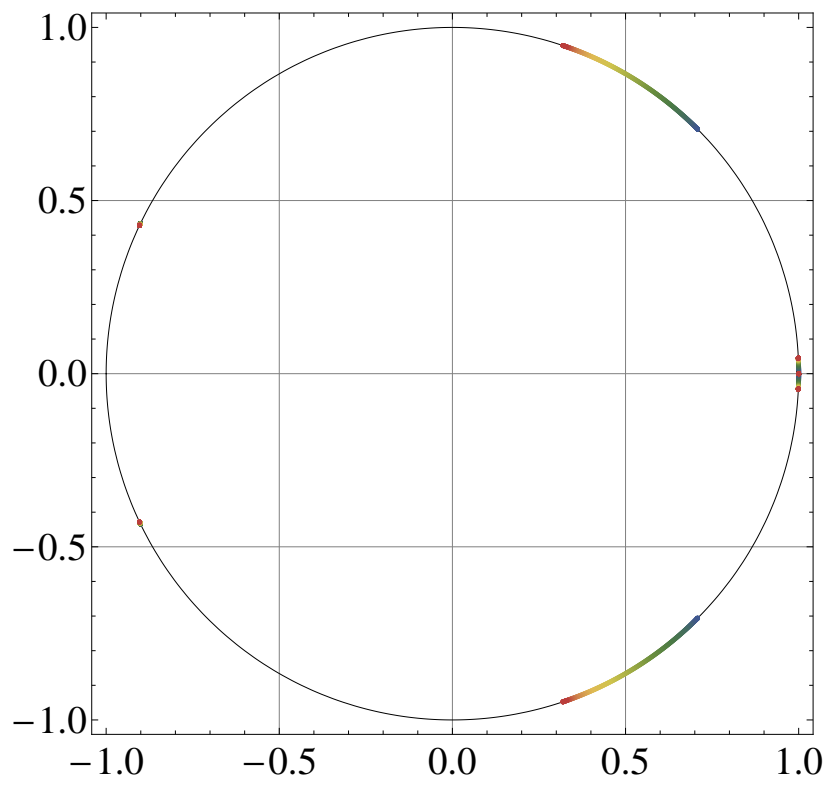

Figure 5: Numerical stability test. The eigenvalues of the amplification matrix. The simulation parameters are: $n_{e^{-}}=3 \cdot 10^{19}\left(1 / m^{3}\right), n_{D}=0.98 n_{e^{-}}\left(1 / m^{3}\right), n_{H}=0.02 n_{e^{-}}\left(1 / m^{3}\right)$. The background magnetic field is uniform and it is equal to $3 \mathrm{~T}$.

validated example shows that the applied numerical scheme is stable for these specific scenario, but it has to be noted that the time step $\Delta t$ must be sufficiently small to satisfy the Courant condition. 


\section{Conclusions}

The finite-difference time-domain(FDTD) method is applied for numerical simulations of the electromagnetic waves in cold magnetized toroidal plasma. The proposed discretization scheme of the Maxwell's equations and the equation of motion has an upper time limit on a time grid step $\Delta t$. This limit should not be exceeded to provide the numerical stability. It is demonstrated that in case of nonmagnetized cold plasma, the maximum stable Courant number of the hybrid method is unity just as for free space, i.e. it coincides with the vacuum Courant condition. For magnetized cold plasma, the stability of the applied scheme is investigated numerically using von Neumann method. It has been found numerically that the plasma resonance area can be problematic for simulations, however the collision frequency term may give the possibility to resolve that problem. It has to be noted that for the ion- cyclotron frequency range in magnetized case a suitable cell size must be carefully chosen to satisfy both the stability condition and the accuracy requirements. The criteria for choosing the size of the grid-mesh may be obtained through dispersion relation analysis. The general conclusion states the necessity of the accurate validation of the numerical stability for considered frequency ranges and expected wave modes.

\section{References}

[1] Cummer S 1997 IEEE Trans. Antennas Propag.392-400 45

[2] Smithe D 2007 Phys. Plasma.056104-7 14

[3] Yu Y, Simpson J 2010 IEEE Trans. Antennas Propag.469-478 58

[4] Tierens W, De Zutter D 2012 Journal of Computational Physics.5144 - 5156231

[5] Moore K 1981 Royal Aircraft Establishment, Technical report.183 205

[6] Richtmyer R, Morton K 1967 Difference Methods for Initial-Value Problems (New York: Wiley)

[7] Yee K 1966 IEEE Trans. Antennas Propag.302-307 14

[8] Woo T, Hagness S 2004 J. Opt. Am. B 221

[9] Sullivan D M 2000 Electromagnetic Simulation Using FDTD Method (New York)

[10] Brambilla M 1998 Kinetic Theory of Plasma Waves (Oxford University Press)

[11] Stix T H 1992 Waves in Plasma (New York)

[12] Hu W, Cummer S 2006 IEEE Trans. Antennas Propag.1513- 152254 